\section{Seeking the soft spots}

Metabolic stability is crucial in allowing drugs to reach therapeutic concentrations, and so highly labile compounds are often filtered out early in the discovery process using metabolic clearance assays. However, such assays do not provide information on the 'soft spots' on the compound that underlie rapid metabolism, limiting rational design to overcome this issue. A paper in the Journal of Medicinal Chemistry now describes how in silico prediction of soft spot location coupled with mass spectrometry (MS) data from high-throughout clearance assays can allow the identification of soft spots much faster and earlier in the drug discovery process.

Data on metabolism mediated by cytochrome P450 (CYP) enzymes - the major contributors to drug metabolism - are typically obtained using high-throughput microsomal assays early in the compound optimization process. Zimmerlin and colleagues set out to use such data by linking it with information derived from a novel tool known as MetaSite, which can predict sites of CYP-dependent metabolism on the basis of computed three-dimensional compound structures and the structure of CYP enzymes. Using information on the most likely soft spots of compounds from MetaSite, the authors' procedure predicts their metabolites in microsomal assays, which are then searched for using sensitive liquid chromatographytandem MS analysis.

To test this procedure, $95 \mathrm{drug}$ candidates from diverse research programmes that showed high clearance in microsomal assays were investigated. Around 55\% of MetaSite's predictions for the top metabolite based on the most likely soft spot were confirmed experimentally, and in another $20 \%$ of cases the top prediction was not detected but the second most likely was found. In a further $9 \%$ of cases, the third most likely metabolite was detected.

So, in this case, the addition of experimental validation of the top three predicted metabolites increases the likelihood of identifying the corresponding soft spot from $55 \%$ to $84 \%$. It therefore seems that incorporating this procedure in early drug discovery could considerably increase the likelihood of focusing optimization efforts most appropriately to improve the metabolic stability of compounds. Furthermore, gathering information on structuremetabolism relationships in this way

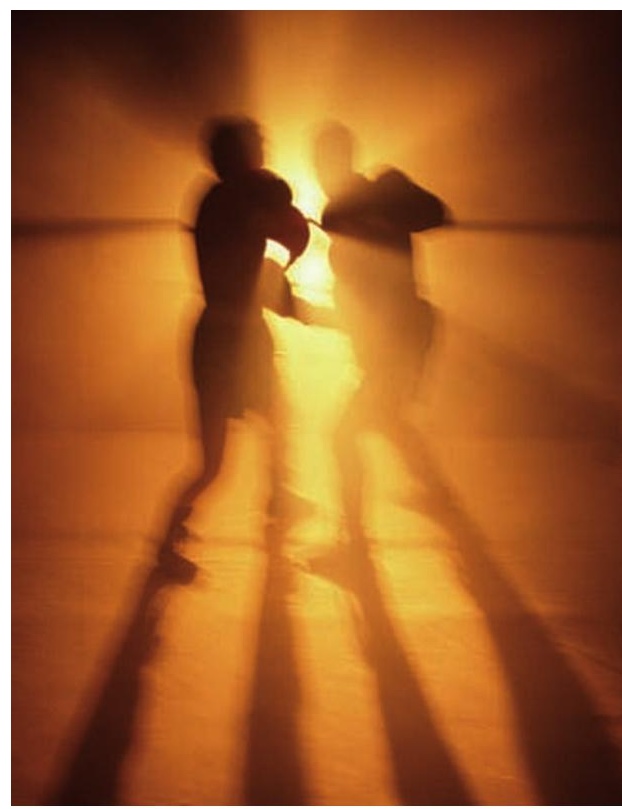

could aid in the development of in silico models that not only identify soft spots with high success rates, but also suggest chemical strategies for stabilization.

Peter Kirkpatrick

ORIGINAL RESEARCH PAPER Trunzer, M. et al. Metabolic soft spot identification and compound optimization in early discovery phases using MetaSite and LC-MS/MS validation. J. Med. Chem. 52, 329-335 (2009) 